\title{
BMI: Good Measure or Fat Chance?
}

\section{Lon Kilgore* and Julien S Baker}

Institute of Clinical Exercise \& Health Science, University of the West of Scotland, Scotland

An increased frequency of obesity is a cross-societal issue that is receiving a tremendous amount of scientific and media attention. Current estimates of obesity range from $35.7 \%$ in the US [1], 26.0\% in the UK [2], to an international low of $3.8 \%$ in Korea [3]. There are myriad proposed explanations proposed for the upwards trend in obesity occurring since 1960 with most explanations being based on correlation and conjecture rather than causality. Despite the lack of causal data, the correlative data is used as a basis for many nations' public health policy. In the UK, the National Health Service proposes that $55 \%$ of the adult population will be obese by 2050 and the calculated annual fiscal health care burden is estimated at $£ 6.6$ to 7.4 billion [4]. This is an alarming statistic and merits attention and there is significant movement within policy makers to respond. But we need to examine the historical aspects of obesity and its measurement in order to avoid alarmism and potential misdirection of national assets.

To more fully understand obesity and our current straits, we need to consider whether the epidemic of obesity is authentic, or is it an artifact of changing social values and constantly changing definitions.

Obesity has not been considered truly unhealthy for the majority of human history, rather it has frequently been a valued survival trait or a symbol of social and economic status. It was not until the early 1900's that obesity began to receive significant attention via its emerging correlation to heart health and cardiac demise. At this point in history, obesity was assessed via the Metropolitan Height and Weight Charts. It must be understood that these charts were designed to be favorable to insurance company profits. Obesity related insurance risk, or the transition from "ideal weight" to "overweight" was set intentionally low in order to expand the population of citizens who would need to pay the higher insurance rates associated with being overweight. It is well known that these tables do not measure obesity, as fat content is not assessed and there are other flaws in these tables that have been identified over the years, but despite the flaws, height and weight tables remain in use in the assessment of obesity nearly a century after they were first developed.

More recently, indirect assessment of subcutaneous fat stores has been proposed as the most effective and accurate means of field measurement of obesity. Generally implemented as a set of caliper based measures that are plugged into a predictive equation, these estimations do account for variations in lean body mass but the resulting data can only be used to estimate an arbitrary range of "healthy" weights for an individual. A defined \% body fat above or below which mortality rate increases was and is not discernable from the existing data.

Body Mass Index has grown in popularity as an easy assessment of obesity. Originally proposed for use as an estimation of body fat by Ancel Keyes in 1972 in large scale population studies (the formula originated about a century and a half earlier) [5]. By the early 1980s the Centers for Disease Control and Prevention and the World Health Organization had adopted BMI as its primary means for assessing overweight status. It is interesting to note that while these organizations largely abandoned height and weight tables due to their problems, BMI is based upon height and weight measures and essentially reflects the same information and same putative level of predictive accuracy as the tables replaced. Regardless, in 1985 the CDC and WHO adopted an arbitrary BMI value, 27.8, as the level above which obesity is diagnosed. At the time this seemed appropriate and reasonable as the mean BMI

\begin{tabular}{|c|c|c|}
\hline Year & per Million Population & BMI \\
\hline 1960 & 55.9 & 25.0 \\
\hline 1970 & 49.3 & 25.4 \\
\hline 1980 & 41.2 & 25.5 \\
\hline 1990 & 32.2 & 26.6 \\
\hline 2000 & 25.8 & 28.0 \\
\hline 2007 & 19.1 & 28.4 \\
\hline
\end{tabular}

Table 1: Cardiovascular Deaths.

among the US population was 25.0 in 1960, 25.4 in 1970, 25.5 in 1980, and 26.6 in 1990 . The 27.8 value represented the upper end of a twotailed normal distribution. Thus the rate of obesity in those decades increased mildly.

But between 1990 and 2000 there was a tremendous increase in the reported obesity rate in the US, from $22.1 \%$ to $29.6 \%$ of the adult population was estimated to be overweight by BMI. This large change does not represent a commensurate increase body size in the US as the average height and weights were $75.8 \mathrm{~kg} / 169.1 \mathrm{~cm}$ and $79.9 \mathrm{~kg} /$ $169.4 \mathrm{~cm}$ respectively [6]. It is a result of the CDC and WHO lowering the critical value representing a positive diagnosis of obesity from 27.8 to 25.0 in this decade inducing the change in diagnostic magnitude. Although the 1990 mean BMI was 26.6 and the 2000 mean was 28.0, the change in measurement standard downwards to 25.0 artificially elevated the rate of increased diagnosis of obesity. This alteration in standard makes epidemiologic predictions based on the new data points unreliable. The population did not magically get fat overnight, the rules changed.

It is also during this decade that the CDC and the ACSM moved obesity from being a secondary risk factor for developing heart disease (another risk elevating factor had to be present for it to increase risk, it couldn't do it by itself) to the level of primary risk factor (independently increases risk of death). This primary risk factor status may have been assigned erroneously because when we simply look at the by-decade mean BMI and the absolute rate of deaths attributed to cardiovascular disease we see that there is a very strong inverse correlation $(r=-0.960)$ between death rate and BMI. As BMI increased over the time period and the rate of death decreased by about $40 \%$, it seems as though obesity does not kill (Table1).

Although this superficially seems to be a strong statement that BMI is not a valid diagnostic tool, it must be considered that advances in pharmacological and surgical therapies for cardiovascular diseases is largely responsible for the increased survival rates noted over the past 50 years. This suggests that BMI, although it is unlikely, may still remain

${ }^{*}$ Corresponding author: Lon Kilgore, Institute of Clinical Exercise \& Health Science, University of the West of Scotland, Hamilton, South Lanarkshire, ML10 6NZ, Scotland, E-mail: Ion.kilgore@uws.ac.uk

Received June 28, 2012; Accepted July 03, 2012; Published July 05, 2012

Citation: Kilgore L, Baker JS (2012) BMI: Good Measure or Fat Chance? J Sports Med Doping Stud 2:e118. doi:10.4172/2161-0673.1000e118

Copyright: @ 2012 Kilgore L, et al. This is an open-access article distributed under the terms of the Creative Commons Attribution License, which permits unrestricted use, distribution, and reproduction in any medium, provided the original author and source are credited. 
a predictor of cardiovascular mortality in some small way. However upon reflection, its utility as a predictive measure is further limited as its precision is quite problematic, with healthy (low mortality) values ranging from 20 to 29.9 [7]. Hardly a picture of a useful measurement tool.

It is germane at this point to discriminate between studies that correlate BMI, bodyweight and body fat to mortality (actual deaths directly attributable to a specific cause) and those that correlate BMI, bodyweight and body fat to risk factors of disease (conditions that may be present at the same time as a disease process). The primary intent of public health initiatives is to prevent unnecessary deaths and its secondary intent is to minimize the occurrence of disease processes in the public. As it is apparent that BMI does not predict cardiovascular mortality, it may still possess some value in assessing risk of developing diseases that do increase mortality through contributions to cardiovascular death.

If we consider one such disease, diabetes mellitus, and its epidemiology in reference to BMI, we see another interesting relationship, rather a lack of relationship, between diabetic death rate and BMI $(\mathrm{r}=0.331)($ Table 2$)$.

So after considering two of the major causes of death globally, and diabetes reportedly acting as a contributor to cardiovascular death, we see that BMI is not an effective tool for predicting mortality from cardiovascular causes, diabetes, or as a pair. This calls into question the practice of using BMI for any diagnostic or prognostic purpose. There is not enough direct evidence to suggest it provides any information of value to clinicians or fitness practitioners.

If we consider other measures of body size and their relationship to mortality (from cardiovascular and diabetic causes) we find nearly identical associations as with BMI:

$$
\begin{array}{ll}
\text { Cardiovascular Death to Bodyweight } & \mathrm{r}=-0.976 \\
\text { Cardiovascular Death to Height } & \mathrm{r}=-0.872 \\
\text { Cardiovascular Death to Waist Circumference } & \mathrm{r}=-0.975
\end{array}
$$

As body size increases, the rate of cardiovascular mortality goes down. This is not intuitive nor is this the information with which the public is presented, they are presented the exact opposite.

Diabetic Death to Bodyweight

$\mathrm{r}=0.293$

Diabetic Death to Height

$\mathrm{r}=0.067$

Diabetic Death to Waist Circumference

\begin{tabular}{|c|c|c|}
\hline Year & per Million Population & BMI \\
\hline 1960 & 2.25 & 25.0 \\
\hline 1970 & 2.43 & 25.4 \\
\hline 1980 & 1.81 & 25.5 \\
\hline 1990 & 2.07 & 26.6 \\
\hline 2000 & 2.52 & 28.0 \\
\hline 2007 & 2.24 & 28.4 \\
\hline
\end{tabular}

Table 2: Diabetes Deaths.

\begin{tabular}{|c|c|c|}
\hline Year & $\begin{array}{c}\text { Cardiovascular Deaths per } \\
\text { Million Population }\end{array}$ & $\begin{array}{c}\text { Percent of Population } \\
\text { Reporting Some Activity }\end{array}$ \\
\hline 1990 & 32.2 & 69.1 \\
\hline 2000 & 25.8 & 72.2 \\
\hline 2007 & 19.1 & 76.1 \\
\hline
\end{tabular}

Table 3: Cardiovascular Deaths- Percent of Population.

These diabetic values present an absent correlation between increased body size and death rates. Again this data, the exact same US government data available to be used to prepare government and media reports, does not support the concepts presented to the public.

Are there any measures that are associated with decreased mortality rates? Yes. Over the past two decades the percentage of US and UK populations who have been physically active has risen. When treated statistically there is an inverse association between an increasingly active population and a decreased death rate from cardiovascular causes (Table 3).

Although the data surrounding physical activity and exercise data is tremendously soft, rife with measurement error and inconsistent definition, the crude correlation is quite robust $(r=0.999)$. Despite this, the simultaneous therapeutic and life extending advances in medicine during the same time period make absolute association or establishment of causality impossible. However, unlike with measures of body dimension, habitual inclusion of activity and exercise into ones activity patterns presents a favorable and easily alterable association one that is available to all populations across all social and economic divisions.

If it is desirable to affect a large scale change in morbidity and mortality, it is incumbent on scientists, clinicians and policy makers to consider how and if the measures used in experimentation are truly appropriate for purpose and are reflective of the desired outcomes. Clear connections between measured variables and exploitable outcomes should be apparent rather than loose and tenuous relationships. Further, it may be more important to more fully explore, given the already substantial investments in pharmacologic and medical interventions, the implementation of innovative exercise training in reduction of morbidity and mortality.

\section{References}

1. Ogden CL, Carroll MD, Kit BK, Flegal, KM (2012) Prevalence of Obesity in the United States, 2009-2010. NCHS Data Brief.

2. McCormick B, Stone I; Corporate Analytical Team (2007) Economic costs of obesity and the case for government intervention. Obes Rev 8: 161-164.

3. NHS Information Centre, Lifestyles Statistics (2012) Statistics on obesity physical activity and diet: England, 2012. National Health Service, London UK.

4. OECD (2011) Health at a Glance 2011: OECD Indicators, OECD Publishing.

5. Keys A, Fidanza F, Karvonen MJ, Kimura N, Taylor HL (1972) Indices of relative weight and obesity. J Chronic Dis 25: 329-343.

6. Ogden CL, Fryar CD, Carroll MD, Flegal KM (2004) Mean body weight, height, and body mass index, United States 1960-2002. Adv Data (347): 1-17.

7. Tamakoshi A, Yatsuya H, Lin Y, Tamakoshi K, Kondo T, et al. (2010) BMI and all-cause mortality among Japanese older adults: findings from the Japan collaborative cohort study. Obesity (Silver Spring) 18: 362-369. 